Conclusion: Although obesity was more frequently reported in RA and PsA patients, age and gender seemed to be the major factors in the occurrence of this difference rather than inflammatory arthritis subgroups. Therefore, when considering obesity as a factor in the registries, for instance biological registries, sex and age should be kept in mind.

REFERENCES:

[1] Højgaard, P., et al., The influence of obesity on response to tumour necrosis factor- $\alpha$ inhibitors in psoriatic arthritis: results from the DANBIO and ICEBIO registries. Rheumatology (Oxford), 2016. 55(12): p. 2191-2199.

[2] Liu, Y., et al., Impact of Obesity on Remission and Disease Activity in Rheumatoid Arthritis: A Systematic Review and Meta-Analysis. Arthritis Care Res (Hoboken), 2017. 69(2): p. 157-165.

[3] Moroni, L., N. Farina, and L. Dagna, Obesity and its role in the management of rheumatoid and psoriatic arthritis. Clin Rheumatol, 2020. 39(4): p. 1039-1047.

[4] Zurita Prada, P.A., et al., Influence of smoking and obesity on treatment response in patients with axial spondyloarthritis: a systematic literature review. Clin Rheumatol, 2020.

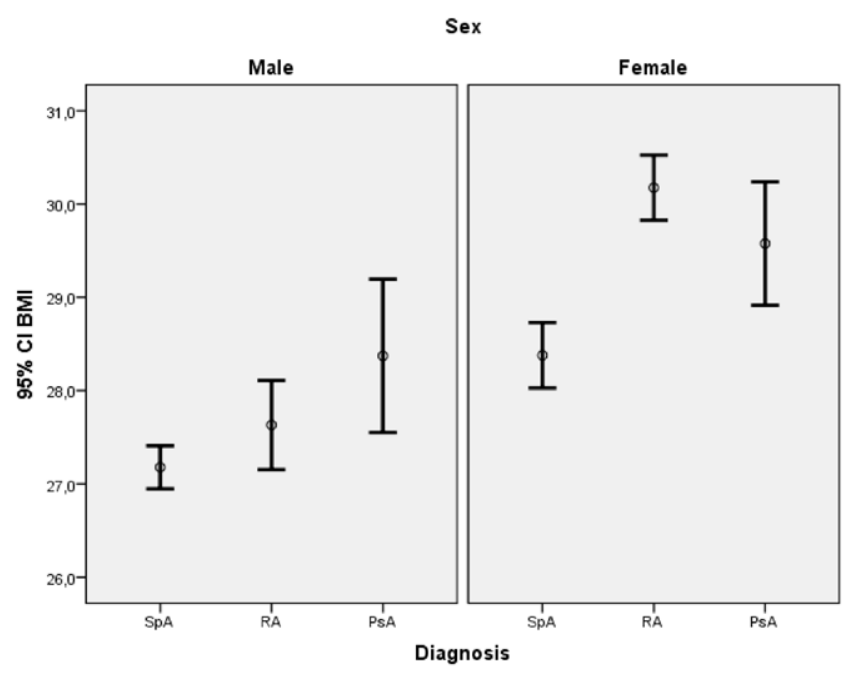

Figure 1. BMI regarding to sex and diseases subtypes

Disclosure of Interests: None declared

DOI: 10.1136/annrheumdis-2021-eular.3312

\section{POS0569 \\ LONG-TERM OUTCOMES OF CHILDREN BORN TO WOMEN WITH RHEUMATOID ARTHRITIS}

N. Calin ${ }^{1}$, A. T. Florescu ${ }^{2}$, F. Bobirca ${ }^{3}$, C. Tataru ${ }^{4}$, I. Ancuta ${ }^{1,2}$, M. Bojinca ${ }^{1,2}$ C. Mihai ${ }^{1,5}$, A. Balanescu ${ }^{1,6}$, A. Musetescu ${ }^{7}$, M. Micu ${ }^{8}$, C. Ancuta ${ }^{9}$, V. Stoica ${ }^{1,2}$, L. Andreoli ${ }^{10,11}$, B. Anca ${ }^{1,2} .^{1}$ Carol Davila University of Medicine and Pharmacy, Rheumatology, Bucharest, Romania; ${ }^{2}$ Cantacuzino Hospital, Internal Medicine and Rheumatology, Bucharest, Romania; ${ }^{3}$ Carol Davila University of Medicine and Pharmacy, Surgery, Bucharest, Romania; ${ }^{4}$ Emergency Hospital Saint Pantelimon, Rheumatology, Focsani, Romania; ${ }^{5}$ Zurich University Hospital, Rheumatology, Zurich, Switzerland; ${ }^{6}$ Sf Maria Clinical Hospital, Internal Medicine and Rheumatology, Bucharest, Romania; ${ }^{7}$ University of Medicine and Pharmacy of Craiova, Rheumatology, Craiova, Romania; ${ }^{8}$ Clinical Rehabilitation Hospital, Rehabilitation II, Cluj-Napoca, Romania; ${ }^{9}$ Grigore T. Popa University of Medicine and Pharmacy, Clinical Hospital of Rehabilitation, Iasi, Romania; ${ }^{10}$ University of Brescia, Clinical and Experimental Sciences, Brescia, Italy;

${ }^{11}$ Spedali Civili Hospital, Rheumatology and Clinical Immunology, Brescia, Italy

Background: Children born to women with rheumatoid arthritis (RA) have increased incidences of adverse neonatal outcomes and a potential excess risk of specific diseases during childhood and adolescence [1]. Further studies aimed at confirming long-term consequences in the offspring are needed.

Objectives: To evaluate whether maternal RA has an impact on the health and developmental outcomes of the offspring.

Methods: A retrospective descriptive study was conducted on data regarding 43 children born to mothers diagnosed with either RA or juvenile idiopathic arthritis (JIA) prior to conception. Participants were recruited from several Clinics of Rheumatology located across Romania. Data on neonatal outcomes, lactation, developmental milestones, childhood illnesses, and hospitalizations was collected using a patient-reported questionnaire completed by maternal participants in 2020 .

Results: Favorable neonatal outcomes were found in $81 \%$ of the participants; however, children of mothers with RA had a higher occurrence of favorable outcomes than those with JIA $(p=0.009)$. Adverse neonatal outcomes reported include the following: small for gestational age (11.6\%), intrauterine growth restriction $(4.65 \%)$, and preterm births $(2.75 \%)$. There were no incidences of congenital malformations. The mean birth weights of offspring born to mothers with RA are higher than those with JIA ( $p=0.00829)$.

While the majority of the children were breastfed $(88.4 \%)$, those who were not breastfed were hospitalized more often than those who were breastfed for any period of time $(p=0.03)$. Mothers who experienced a postpartum flare up within the first 4 months breastfed their children significantly less than those who did not have a flare up (14.62 versus 48 weeks, $p=0.00011$ ).

The mean age of the children at inclusion was $7.6 \pm 5.5$ years.

Developmental milestones were considered to be within the expected average limits, except for an increased incidence of delayed speech (12\%).

$51.2 \%$ of the children were hospitalized at some point during their childhood, with viral enterocolitis, tonsilitis, bronchiolitis, and pneumonia being the most frequently encountered reasons for admission. Surprisingly, children with favorable neonatal outcomes were hospitalized more often than those with unfavorable outcomes $(p=0.0000436)$. Additionally, children born to mothers under the age of 35 were hospitalized more often than those over $35(p=0.02)$.

$77.3 \%$ of offspring experienced recurrent ear/nose/throat (ENT) infections, $55.8 \%$ had allergies, and $39.5 \%$ were diagnosed with atopic dermatitis. Childhood allergies were strongly associated with an increased incidence of atopic dermatitis ( $p$ $=0.0000585)$, as was a concurrent maternal thrombophilia diagnosis $(p=0.03)$ Of the remaining childhood afflictions that were assessed asthma/chronic respiratory diseases $(4.65 \%)$, juvenile idiopathic arthritis $(2.33 \%)$, ulcerative colitis $(2.33 \%)$, diabetes, anxiety/personality disorders, thyroid diseases, febrile seizures, and epilepsy did not yield statistically significant results.

Conclusion: Maternal RA was found to be associated with increased incidences of adverse neonatal outcomes, childhood hospitalizations, recurrent ENT infections, allergies, and atopic dermatitis. However, overall health outcomes of offspring did not show alarmingly significant excess morbidities.

\section{REFERENCES:}

[1] Rom AL, Wu CS, Olsen J, et al. Parental rheumatoid arthritis and long-term child morbidity: a nationwide cohort study. Annals of the Rheumatic Diseases. 2016;75(10):1831-1837. doi:10.1136/annrheumdis-2015-208072

Disclosure of Interests: None declared

DOI: 10.1136/annrheumdis-2021-eular.3322

\section{POS0570 ASSOCIATION OF BODY FAT MASS AND ITS DISTRIBUTION WITH DISEASE ACTIVITY, PAIN AND DISABILITY IN RHEUMATOID ARTHRITIS}

O. Lamkhanat ${ }^{1}$, H. Azzouzi ${ }^{1}$, I. Linda ${ }^{1} .{ }^{1}$ Mohammed VI University Hospital, Mohammed I University, Faculty of Medicine, Rheumatology, Oujda, Morocco

Background: Rheumatoid arthritis (RA) and body composition are closely related. Recent studies have found a significant association between fat mass and disease activity and disability in RA [1]

Objectives: We aimed to study the association between body fat mass and its distribution with disease activity, disability, and pain in RA patients.

Methods: This is a cross-sectional study of patients with RA diagnosis according to ACR-EULAR 2010 classification recruited from first January 2021. Those with prior cancer, hyperparathyroidism, hyperthyroidism, diabetes, chronic kidney disease, and cirrhosis were excluded. Body fat mass (BFM) and its distribution (gynoid (GFM), android (AFM), visceral (VFM), limbs (LFM), trunk (TFM)) were measured with dual-energy X-ray absorptiometry (Hologic, Horizon QDR $囚$ ). Clinical data and laboratory tests of the same day of the DXA scan were analyzed. The associations between BFM and its distribution with disease activity score (DAS28CRP), pain visual analogue scale (VAS), and disability measured by health assessment questionnaire (HAQ) were explored. Obesity was defined as a body mass index $(\mathrm{BMI}) \geq$ of $25 \mathrm{~kg} / \mathrm{m}^{2}$. Our statistical analysis was based on descriptive study, comparisons and linear regressions using SPSS 20.

Results: It is about 69 RA patients. Their mean age was $49.86 \pm 14.33$ years mean DAS28CRP was $2.56 \pm 1.27$, and mean disease duration was 14.84 \pm 10.99 years. Sixty-two (89.9\%) were women. The mean BMI was $26.46 \pm$ $5.26 \mathrm{~kg} / \mathrm{m}^{2}$, and 41 patients were obese $(59.4 \%)$. Compared with non-obese patients, obese patients had a higher $\mathrm{C}$-reactive protein $(\mathrm{p}=0.03)$. DAS$28 \mathrm{CRP}$ was higher in obese patients $(2.77 \pm 1.41$ vs $2.25 \pm 0.97)$ but did not reach significance $(p=0.07)$. We did not find any difference between the two groups regarding pain and disability. In univariate regression analysis, the LFM was positively associated with disease activity $(p=0.001 ; \beta=0.38)$, pain $(p=0.001 ; \beta=0.38)$ and disability $(p=0.007 ; \beta=0.32)$. Adjusted on BMI, LDL cholesterol, triglyceride, cumulative dose of corticosteroid, disease and corticosteroid duration, menopause duration, dose and duration of methotrexate, we found a significant association between LFM, disability $(p=0.02$ $\beta=0.51)$, disease activity $(p=0.02 ; \beta=0.54)$ and pain $(p=0.009 ; \beta=0.57)$. However, we had no association between disease activity, pain, and disability with BFM and the other components.

Conclusion: Limbs fat mass was significantly associated with the activity, disability, and pain in RA patients. 
REFERENCES:

[1] Kyeong Min Son, Seong Hun Kang, Young II Seo, Hyun Ah Kim. Association of body composition with disease activity and disability in rheumatoid arthritis. Korean J Intern Med. 2021 Jan;36(1):214-222.

Disclosure of Interests: None declared

DOI: 10.1136/annrheumdis-2021-eular.3337

\section{POS0571 NON-OBESE RHEUMATOID ARTHRITIS PATIENTS WITH LOW LOW-DENSITY LIPOPROTEIN HAVE GREATER CORONARY ATHEROSCLEROSIS BURDEN, PLAQUE PROGRESSION AND CARDIOVASCULAR EVENT RISK}

G. Karpouzas ${ }^{1}$, S. Ormseth ${ }^{1}$, E. Hernandez ${ }^{1}$, M. Budoff ${ }^{2}{ }^{1}$ The Lundquist Institute, Rheumatology, Torrance, United States of America; ${ }^{2}$ The Lundquist Institute, Cardiology, Torrance, United States of America

Background: Rheumatoid arthritis (RA) patients with low body weight incur higher mortality than obese patients. Paradoxically, RA patients in the lowest low-density lipoprotein group ( $\mathrm{LDL}<70 \mathrm{mg} / \mathrm{dl}$ ) may also experience higher cardiovascular disease (CVD) risk.

Objectives: We here explored whether abdominal obesity (waist-to-height ratio $>0.58$ in females and $>0.63$ in males) might moderate the effect of low LDL $(<70 \mathrm{mg} / \mathrm{dl})$ on coronary atherosclerosis burden, progression and long-term CVD risk in RA.

Methods: One hundred fifty patients without symptoms or diagnosis of CVD underwent coronary computed tomography angiography. Plaque progression was evaluated $6.9 \pm 0.3$ years later in 101 patients. Coronary artery calcium, number of segments with plaque (segment involvement score), and extensive ( $>4$ segments with plaque) or obstructive disease ( $>50 \%$ stenosis) were assessed. CVD events were prospectively recorded, including cardiac death, myocardial infarction, unstable angina, revascularization, stroke, claudication, and heart failure hospitalization over $6.0 \pm 2.4$ years of follow-up. Lipoprotein classes were directly measured. Oxidized LDL (oxLDL) was assessed with monoclonal antibody E06. Adjusted robust linear regression evaluated interactions between abdominal obesity and LDL groups on plaque outcomes. Per segment, adjusted robust logistic regression models explored obesity $\mathrm{x}$ LDL group interactions on new plaque formation and stenotic progression of prevalent plaques. Robust Cox regression models stratified by abdominal obesity evaluated the effect of LDL group ( $<70 \mathrm{vs.}>70 \mathrm{mg} / \mathrm{dl}$ ) on CVD events.

Results: Non-obese patients with low LDL had the highest plaque burden (Figure $1 \mathrm{~A}$, all $\mathrm{p}<0.02$ ). Obesity moderated the effect of $\mathrm{LDL}$ on likelihood of extensive/obstructive disease $(P$ for interaction $=0.061)$; specifically, $\mathrm{LDL}<70$ associated with an increased likelihood of extensive/obstructive plaque in non-obese (adjusted OR 4.75 [95\% Cl 1.18-19.07], $P=0.028$ ) but not obese patients (adjusted OR $1.55[95 \% \mathrm{Cl} 0.39-6.08], P=0.532$ ). No differences in disease activity or inflammatory markers were seen across groups. Compared to LDL $>70 \mathrm{mg} / \mathrm{dl}$, low LDL predicted an increased likelihood of high oxLDL (>median) in non-obese patients (adjusted OR 5.10 [95\% Cl 1.46-17.75], $P=0.011$ ) but not obese patients (adjusted OR 0.50 [95\% Cl 0.11-2.21], $P=0.36$, Figure 1B). Obesity further moderated the effect of LDL on likelihood of future development of plaque ( $P$ for interaction $=0.002)$ and increased stenotic severity of existing plaques $(P$ for interaction $=0.040$ ); in non-obese patients, low LDL associated with a greater likelihood of new plaque forming in segments without baseline plaque (adjusted OR 4.68 [95\% Cl 2.26-9.66]) and worsening stenotic severity in segments with prevalent plaque (OR 5.35 [95\% $\mathrm{Cl} 1.62-17.67])$. This was not observed in obese patients (Figure 1C). Notably, in non-obese patients, low LDL associated with higher CVD event risk compared to those with LDL>70 mg/dl (HR 7.94 [95\% $\mathrm{Cl} 1.52-41.36], P=0.015)$. This was not the case in obese patients (HR 0.32 [95\% Cl 0.04-2.40], $P=0.27$, Figure 1D).

Conclusion: In non-obese RA patients, $\mathrm{LDL}<70 \mathrm{mg} / \mathrm{dl}$ may reflect higher $\mathrm{LDL}$ oxidation and was associated with higher baseline coronary atherosclerosis burden, new plaque formation, stenotic plaque progression and greater CVD risk than LDL>70 mg/dl.

Disclosure of Interests: George Karpouzas Speakers bureau: Sanofi/ Genzyme/ Regeneron, Consultant of: Sanofi/ Genzyme/ Regeneron, Grant/research support from: Pfizer, Sarah Ormseth: None declared, Elizabeth Hernandez: None declared, Matthew Budoff Consultant of: Pfizer

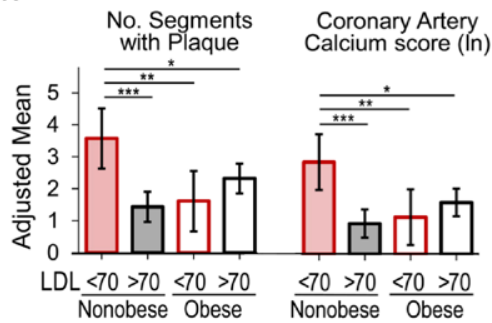

C

\begin{tabular}{|c|c|c|c|}
\hline Outcome & Adjusted & d OR $(95 \% \mathrm{Cl})$ & $P$ value \\
\hline \multicolumn{4}{|c|}{ Increased stenosis } \\
\hline Obese & & $1.1(0.1-15.2)$ & 0.958 \\
\hline Non-obese & $\rightarrow-$ & $5.4(1.6-0.17 .7)$ & 0.006 \\
\hline \multicolumn{4}{|l|}{ New plaque } \\
\hline Obese & $\rightarrow$ & $0.7(0.2-2.5)$ & 0.562 \\
\hline Non-obese & $\rightarrow$ & $4.7(2.3-9.7)$ & $<0.001$ \\
\hline \multicolumn{4}{|c|}{$\begin{array}{lllll}0.01 & 0.1 & 1.0 & 10 & 100\end{array}$} \\
\hline $\begin{array}{l}\text { Highe } \\
\text { LDL>7 }\end{array}$ & $\begin{array}{l}\text { rfor Higher } \\
70 \quad \text { LDL }\end{array}$ & & \\
\hline
\end{tabular}

Figure 1 (A) Mean number of coronary segments plaque and log-transformed coronary artery calcium score across LDL/obesity groups, adjusted for age, gender, diabetes, and statin use. (B) Percent patients with elevated (>median) oxidized LDL (OxLDL) across LDL (B) adjusted for age, gender, hypertension, diabetes, and statin use. (C) Moderating effects of obesity on the association between low LDL and worsening stenosis of existing plaques and new plaque formation in coronary segments without baseline plaque, adjusted for age, time between (D) The in duration. New plaque formation modeladditionaly

$* p<0.050,{ }^{* *} p<0.010,{ }^{* * *} p<0.001$.

DOI: 10.1136/annrheumdis-2021-eular.3419

\section{POS0572 THE ASSOCIATED FACTORS OF ULTRASONOGRAPHIC TENOSYNOVITIS IN PATIENTS WITH RHEUMATOID ARTHRITIS IN REMISSION}

R. Fakhfakh ${ }^{1}$, N. El Amri ${ }^{1}$, K. Baccouche ${ }^{1}$, H. Zeglaoui ${ }^{1}$, E. Bouajina ${ }^{1} .{ }^{1}$ Farhat Hached Hospital, Rheumatology, Sousse, Tunisia

Background: Tenosynovitis is a common manifestation of rheumatoid arthritis (RA), but, is difficult to assess appropriately by clinical examination. In clinical remission, musculoskeletal ultrasound (MSUS) showed a predictive value of tenosynovitis for flares and joint destruction in RA (1).

Objectives: To assess the prevalence, the characteristics, and the factors associated with tenosynovitis in ultrasound examination of patients with RA in clinical remission.

Methods: A Cross-sectional study including patients with RA in clinical remission (DAS28 ESR $\leq 2.6$ ) for at least 6 months. MSUS of 20 tendons (in wrists, hands and ankles) was performed in mode $\mathrm{B}$ and power Doppler (PD). Tenosynovitis was defined and scored according to the Outcome Measures in Rheumatology Clinical Trials (OMERACT). A total score for the tenosynovitis in B-mode (TBI) and PD (TDI) was calculated by summing the grades for each patient. SDAI and CDAI were calculated. Functional disability was assessed by the Health Assessment Questionnaire (HAQ). For the radiographic evaluations, we used the Van der Heijde score.

Results: Thirty-seven patients were enrolled, the mean age was 54.2 years \pm 12.7 years and the sex ratio was 0.37 . The mean disease duration was 8.1 years \pm 5.1 . The mean remission duration was 36.5 months \pm 32.7 . The prevalence of tenosynovitis was $40.5 \%$ in B-mode and $13.5 \%$ for PD. The most affected tendons were: extensor carpi ulnaris (B-mode: $21.6 \%$, PD: $8.1 \%$ ), tibialis posterior (B-mode: 10\%, PD: $2.7 \%$ ), peroneal (B-mode: $8.1 \%$, PD: $2.7 \%$ ), flexor digitorum superficialis and profundus (B-mode: $5.4 \%, \mathrm{PD}: 0 \%$ ) and extensor digitorum (B-mode: 2.7\%, PD: 2.7\%). Tenosynovitis was most frequently grade1 in B-mode $(32.4 \%)$ and grade 2 in PD $(10.8 \%)$. Grade 3 was identified only in B-mode in the peroneal tendons. The mean of TBI was $7.1 \pm 5.3$ and the mean of TDI was 2.2. \pm 3.1 . We found a significant association between remission duration and $P D$ tenosynovitis (mean: 16.2 vs 39.7 months, $p=0.05$ ). Tenosynovitis, in $B$-mode and PD, weren't associated with the swollen joints, the tender joints, the patient 